\title{
Bridging Insights: on the Efficacy of Traditional Chinese Medicine in Treating COVID-19 Patients
}

\author{
Ying Bian ${ }^{1 *}$, Yonghua Zhao' ${ }^{1}$, Yitao Wang ${ }^{1}$ and Xueni Bian ${ }^{2}$ \\ ${ }^{1}$ State Key Laboratory of Quality Research in Chinese Medicine, Institute of Chinese Medical Sciences, University of Macau, Macau SAR, China \\ ${ }^{2}$ Department of Life Sciences, Imperial College London, UK
}

*Corresponding author: Ying Bian, State Key Laboratory of Quality Research in Chinese Medicine, Institute of Chinese Medical Sciences, University of Macau, Macau SAR, China.

Received Date: June 09, 2020

Published Date: June 22, 2020

\section{Introduction}

Since the outbreak of Coronavirus disease (COVID-19 hereafter), a generous amount of resources and labour -- from across many different regions of China -- have been dedicated towards better integration of Traditional Chinese and Western medicine. Concerted efforts to unite the two schools were exerted upon all stages of treatment: from diagnosis, to prescription, to proprietary procedures. These measures have effectively leveraged the prowess of traditional Chinese to raise rates of recovery and reduce that of mortality. Not only does the State clearly advocate for strategies which "adhere to the merging of Traditional Chinese and western medicinal concepts; strengthen scientific research; accelerate screening for high clinical-efficacy drugs", practice has shown -- in community measures as well as in clinical treatments -- that equal emphasis on Chinese and Western medicine is a step towards optimising treatment efficacy. Chinese history tells of many past epidemics from which the use of traditional Chinese medicines has accumulated a wealth of experience: Japanese encephalitis in the 1960s, haemorrhagic fever in the 1990s, and SARS in 2003.

The contribution of Chinese medicine to COVID-19 treatment has become a highlight of Wuhan's epidemic prevention and control. Chinese clinical data shows that by the end of March, of those diagnosed with COVID-19, as much as 74,187 (91.5\%) had used traditional Chinese medicine. Of diagnosed patients who had used traditional Chinese medicine, 61,449 (90.6\%) were from Hubei Province. Clinical data shows that the total effective rate of traditional Chinese medicine is more than $90 \%$ [1].
As we stand, currently, in the early stages of a global pandemic, amongst the absence of specialised drugs and vaccines, expertise and efficacy of traditional Chinese medicine in COVID-19 treatment can be summarised as follows. At present, a double-triad of "three medicines and three formulae" have been screened out, with marked effect in treatment. The first triad of medicines being:

Our qualitative study of 17 randomly selected sermons indicates that digital church communication creates a viable messaging platform for churches during social distancing.

- Jinhua Qinggan granule (金花清感颗粒)

- Lianhua Qingwen capsule (莲花清盒胶囊)

- XueBijing injection (血必净注射液)

And the second triad of formulae being:

- $\quad$ Lung cleansing \& detoxifying decoction (清肺排毒汤)

- Huashibaidu Formula (化湿败毒方)

- Xuanfeibaidu Granule (宣肺败毒顆粒)

Why is Chinese Medicine Effective: Reuniting Tradition Theories with Pathology

Much of the literacy with regards to traditional Chinese medicinal concepts still exists in legacy terms: the result of combined erudition from thousands of years of dedication and scholarship. However, dated concepts may as well benefit modern times. As an example, complexities due to (and mishandling of) viscous fluids 
remain one of the important causes of death in critically ill patients. Autopsies showed grey-white viscid fluid in the lungs, white foam-mucus in the tracheal cavity, and films of gelatinous-mucus adhered to bronchial walls, causing subsequent airway blockage, hypoxia, and death by respiratory failure. Clinical treatments show that blind use of ventilation-devices lead to pressurised air pushing mucus deeper into the lungs, exacerbating the patient's hypoxic state. In Chinese medicine, the formalised term for viscous liquids is "phlegm-dampness" (痰湿) [2]. Relief of obstruction within airways is legitimised with its own vocabulary and handled with priority. The Lung cleansing \& detoxifying decoction (清肺排毒汤) -- LCD-decoction hereafter -- is such a prescription for "phlegm-dampness", with efficacy hinging on promotion of water-reabsorption and urination (processes described with respective archaic, parallel concepts: "Qi transformation" and "drainage").

LCD-decoction dates back to the ancient Chinese medical sage Zhang Zhongjing (150-215 AD). It constitutes four recipes: Maxing Ganshi decoction (麻杏甘石汤), Minor Bupleurum decoction ( 小柴胡汤), Five-Ingredient Formula with Poria (五苓散), and Belamcanda-Ephedra decoction (射干麻黄汤). LCD-decoction was used in China to treat COVID-19 with an efficacy of more than $90 \%$, primarily in early intervention to reduce the progression of mild symptoms to severe conditions [3]. In 10 provinces across the country (Hubei Province is not included here), 1,263 diagnosed patients were admitted to 66 designated hospitals, of which 1,214 (96.12\%) were cured and discharged. Of the 57 critically ill patients who were treated with LCD-decoction, 42 (73.7\%) were cured and discharged. Contrast-imaging of the lungs showed that after taking LCD-decoction for two courses (six days), lung lesions of 53 patients (93\%) showed significant degrees of reduction and absorption. Therefore, LCD-decoction contributes significantly to in hindering COVID-19 progression from mild to severe [4].

\section{Technological Advances Promote the Continued Development of Chinese Medicine}

Since the treatment of SARS in 2003, Chinese medicine has made immense progress in terms of scientific and technological advances. Within the 20-year span from 2000 to 2019, a total of 114 projects from the Chinese medicine community have won the National Science and Technology Award [5], amongst whom is the internationally celebrated Tu Youyou, from the Chinese Academy of Medical Sciences, who achieved the highest prestige in science and technology, having received the 2015 Nobel Prize in Physiology and Medicine for her artemisinin research. Therefore, with the assistance of advancing technology, traditional Chinese medicine has indeed progressed to anchor itself on firmer scientific grounds. Immediately after the COVID-19 outbreak, prescription of treatment using traditional Chinese medicine was formulated. To establish initial direction of treatment, he traditional Chinese medicine approach sought for symptom-based clinical investigations, having preliminarily determined that the manifestation of the virus is clinically congruent with that of a "Humid Epidemic" (" 湿毒疫”) [6]. Coronaviruses have been reported since 2006, with abundant presence in existing literature [7]. China used big data to screen more than 40,000 traditional Chinese medicines. There currently exist more than 60,000 component libraries, establishing the chemical structure and active compound of Chinese medicines. Symptom-based clinical observations were used to formulate the Xuanfeibaidu Granule (宣肺败毒颗粒), which consisted of four sub-formulae: Maxing Ganshi decoction (麻杏甘石汤), Maxing Yigan decoction (麻杏薏甘汤), Qianjin Weijing decoction (千金苇 茎汤), and the Lepidium / Descurainiae and Jujube Lung-Draining Decoction (葶芳大查泻肺汤). Data from component library showed that there are two base materials that may be active on COVID-19. (1) Polygonum (Polygonum Cuspidatum Sieb. Et. Zucc. or 虎杖), in which is contained polydatin (虎杖苷) which has a strong inhibitory effect on coronaviruses. (2) Verbena officinalis L. (马鞭草) show strong inhibitory activity against respiratory damage and microthromboses related to coronaviruses, particularly of fine airways. Xuanfei Baidu Granules is thus the combined effort of persevering, ancient scholarship in Chinese medicine and modern, 21st century technology. Through a comparative observation of 120 cases from the Hubei Integrated Traditional Chinese and Western Medicine Hospital, it was found that this formula has shown good effect in improving COVID-19 symptoms (including fever, cough, asthma, and fatigue). It has significant effect on inhibiting C-reactive protein (by 75\%) and raising lymphocyte count (by 17\%). Another 280 cases were observed in Wuhan Jiangxia's mobile-cabin hospitals (frequenting international headlines as "Fang-Cang's") and none of the cases had later progressed into severe conditions.

\section{Rethinking Chinese Medicine: Plight of Tradition Within China}

Within China itself, the use of Chinese medicine is not without resistance, nor doubt with regards to its efficacy. In the COVID-19 pandemic, the distrust focussed on the use of traditional Chinese medicinal injections in treating patients. The suite of injections themselves, as recommended in the COVID-19 National Diagnosis and Treatment Plan, has been thoroughly evaluated in clinical marketing, and has proven track record -- with years of clinical practise -- in efficacy and safety. At present, one-third of Chinese medicine injections should rightly be eliminated. Persisting scepticism about Chinese medicine injections mainly originate from these. However, the use of legitimate injections should not be discredited, especially in critically ill patients, where timely intervention of injections can play a significant role. For those patients with low oxygenation levels and large fluctuations in blood oxygen saturation level, ShengMai injection (生脉注射液) and Shen-Mai injection (参麦注射液) are used. After one to two days, the patient's blood oxygen saturation approaches stability. After three to four days, oxygenation index increases [8]. For severe patients under ongoing cytokine storm, XueBiJing injections (血必净注射液) delay the development of the disease. For those with absorption hindered, due to lung in- 
fections, Reduning injection (加注热毒宁) and Tanreqing injection (痰热清注射液) interact with antibiotics to achieve a synergistic effect [9]. This suite of hybrid strategies, where treatments are hybridised between Chinese and Western medical consultations, and severe patients are considered for Chinese medicine injection as early as possible, is implemented in Wuhan Jinyintan Hospital, Wuhan Pulmonary Hospital, and Wuhan Union Medical College Hospital.

\section{Developmental Prospects}

Chinese medicine -- in its theory and implementation -- has withstood the test of plagues time and time again in the history of China. Cumulative experience garnered is certainly worthy of consideration and merit, with clinical value still relevant today. When it comes to COVID-19 diagnosis and treatment, there is no necessary conflict between the fact that "specialised disease requires specialised prescriptions" and the equally valid "one person requires one formula". Symptom-focussed approaches and disease-focussed approaches can be organic combined so that different treatment strategies can be adopted as is tailored to state of the patient. During the height of the pandemic, traditional Chinese medicines participated extensively in the clinical treatment of COVID-19. More than 4,000 traditional Chinese medicine staff participated on the frontline: from establishing central wards, to the funding and construction of Jiangxia Mobile Cabin Hospitals, to designated hospitals (such as Hubei Integrated Traditional Chinese and Western Medicine Hospital, Wuhan City Chinese Medicine Hospital). Patients received systematic and standardised Chinese medicine treatment, which has achieved good results. This experience within China has shown that: for quarantining suspected cases, fevers, diagnosed cases, and their close contact, Chinese medicine decoctions have the effect of differentiating the population and controlling disease spread; In Fang-Cangs, we see Chinese medicine taking the main role in treatment for mild cases; in severe cases, Chinese medicine play adjuvant but significant roles in alleviating symptoms and accelerating recovery [10]. The experience gained by China should be correctly understood by the international academic community and WHO. In the context of global public health governance, it is a certifiably worthwhile effort to pay attention to and give deserved credit to Chinese solutions. The Chinese have achieved an effective combination of Chinese and Western medicine in clinical treatment, laying a foundation for future construction of a medical service system with Chinese characteristics.

\section{International Outlook}

On March 23, 2020, a spokesperson from the National Administration of Traditional Chinese Medicine stated that the treatment plan implemented in China has been factually proven to be the correct strategy. China is willing to offer Chinese medicine support, alongside relevant expertise, to various countries. Relevant Chinese organizations and institutions have donated patented Chinese medicine, acupuncture needles, and other medicinal resources to countries and regions such as Italy, France, Hong Kong, and Ma- cau S.A.R. Active collaboration with WHO has been initiated, with plans of Chinese medicine treatment being made available in quality English. China also offered technical solutions through remote audio-visual exchanges, sharing treatment expertise with Japan, South Korea, Italy, the United States, Iran, Singapore and others. By March 23, 2020, China has already dispatched 100,000 boxes of Chinese patent medicine to Italy, with another 100,000 boxes to be dispatched in the distant future, upon the request from Italy that more is needed [11]. In the current COVID-19 epidemic, one can see that the integration of traditional Chinese and Western medicine takes shape without redundancy, as the juxtaposition of roles are mutually non-conflicting: there is marked efficacy in traditional Chinese medicines in the progression of cases from mild to severe, with important role in speeding up recovery. This -- particularly with purgative drugs -- in concerted effort with Western medicinal life support technology contribute to reducing case fatality rate. In the absence of special drugs and vaccines, as we have currently with regards to COVID-19, the Chinese expertise and experience comes from having traditional treatments summarised, ancient classics revisited, and altogether utilised in combination with modern clinical practice. In this manner, China hybridised traditional Chinese medicine with Western medicine to achieve efficacy in treatment. As of 3rd March 2020, the 7th edition of "Diagnosis and Treatment Protocol for Novel Coronavirus Pneumonia" has been published [12]. The protocol stands in advocacy for a select batch of Chinese medicine, among which is the Lung cleansing \& detoxifying decoction, being appraised for playing an important role in preventing progression of cases from mild to severe, and greatly reducing the mortality rate.

Traditional Chinese medicine, in its theory, diagnosis, and treatment, sediments from a place of profound time-tested expertise. If understood in a holistic, non-judgmental manner, the crystallised erudition of Chinese medicine, bourn from thousands of years of scholarship and craftsmanship, could be better distilled and integrated for modern-relevance, and utilised to bring a new dawn to patients suffering under the COVID-19 pandemic.

\section{Authors Contributions}

XB drafted the manuscript. LY analysed the data and reviewed the manuscript critically. YB designed the project and did the critical review of manuscript.

\section{Funding}

This work was supported by University of Macau Research Funding support (Grant number MYRG2019-00044-ICMS).

\section{Conflict of Interest}

The authors declare no competing interest

\section{References}

1. (2020) Decoding: Chinese medicine was the only theme for the release conference of the State Council Information Office of the People's Republic of China. 
2. Ren, JL, Zhang AH, Wang XJ (2020) Traditional Chinese Medicine for COVID-19 Treatment. Pharmacological Research. 155: 104743.

3. Li RF, Hou YL, Huang JC, Pan WQ, Ma QH, et al. (2020) Lianhuaqingwen exerts anti-viral and anti-inflammatory activity against novel coronavirus (SARS-CoV-2). Pharmacological Research 156: 104761.

4. China Net of Traditional Chinese Medicine (CNTCM).

5. Guide Web for Chinese Trade in Services (GWCTS).

6. Wang YL, Xiao GX, He S, Liu XY, Zhu L, et al. (2020) Protection against acute cerebral ischemia/reperfusion injury by QiShenYiQi via neuroinflammatory network mobilization. Biomedicine \& Pharmacotherapy 125: 10945.

7. Duan ZP, Jia ZH, Zhang J, Liu S, Chen Yet al. (2011) Natural herbal medicine Lianhuaqingwen capsule anti-influenza A (H1N1) trial: a randomized, double blind, positive controlled clinical trial. Chin Med J (Engl) 124(18): 2925-2933.

8. Han JY, Li Q Ma ZZ, Fan JY (2017) Effects and mechanisms of compound Chinese medicine and major ingredients on microcirculatory dysfunction and organ injury induced by ischemia/reperfusion. Pharmacol Ther 177: $146-173$.
9. Sun K, Fan JY, Han JY (2015) Ameliorating effects of traditional Chinese medicine preparation, Chinese materia medica and active compounds on ischemia/reperfusion induced cerebral microcirculatory disturbances and neuron damage. Acta Pharm Sin B 5(1): 8-24.

10. Yao KT, Liu MY, Li X, Huang JH, Cai HB (2020) Retrospective Clinical Analysis on Treatment of Novel Coronavirus-infected Pneumonia with Traditional Chinese Medicine Lianhua Qingwen. Chinese Journal of Experimental Traditional Medical Formulae 26(11): 8-12.

11. (2020) New perspective of traditional Chinese Medicine in the context of the global (COVID-19) pandemic. Xinhuanet.

12. National Health Commission of the People's Republic of China (NHCPRC). National Administration of Traditional Chinese Medicine (NATCM). The notice of delivering the Diagnostic and Treatment Programmes for COVID-19. 